\title{
Measurable Riesz spaces
}

\author{
Krasikova I. ${ }^{1}$, Pliev M. ${ }^{2,3}$, Popov M. ${ }^{4,5}$ \\ We study measurable elements of a Riesz space $E$, i.e. elements $e \in E \backslash\{0\}$ for which the \\ Boolean algebra $\mathfrak{F}_{e}$ of fragments of $e$ is measurable. In particular, we prove that the set $E_{\text {meas }}$ of all \\ measurable elements of a Riesz space $E$ with the principal projection property together with zero is \\ a $\sigma$-ideal of $E$. Another result asserts that, for a Riesz space $E$ with the principal projection property \\ the following assertions are equivalent. \\ (1) The Boolean algebra $\mathcal{U}$ of bands of $E$ is measurable. \\ (2) $E_{\text {meas }}=E$ and $E$ satisfies the countable chain condition. \\ (3) $E$ can be embedded as an order dense subspace of $L_{0}(\mu)$ for some probability measure $\mu$. \\ Key words and phrases: vector lattice, Riesz space, Boolean algebra of bands.

\footnotetext{
${ }^{1}$ Zaporizhzhya National University, 66 Zhukovskoho str., 69002, Zaporizhzhya, Ukraine

2 Southern Mathematical Institute of the Russian Academy of Sciences, 22 Markusa str., 362027, Vladikavkaz, Russia

${ }^{3}$ North-Ossetian State University, 44-46 Vatutina str., 362025 Vladikavkaz, Russia

${ }^{4}$ Institute of Exact and Technical Sciences, Pomeranian University in Słupsk, 22A Arciszewskiego str., 76-200, Słupsk, Poland

${ }^{5}$ Vasyl Stefanyk Precarpathian National University, 57 Shevchenka str., 76018, Ivano-Frankivsk, Ukraine

E-mail: irynazpukr@gmail.com (Krasikova I.), plimarat@yandex.ru (Pliev M.), misham.popovegmail.com (Popov M.)
}

\section{Introduction}

In recent paper [8], the third named author used the term "measurable Riesz space" in the following sense: a Riesz space $E$ is said to be measurable provided for every $e \in E^{+}$the measurable Boolean algebra $\mathfrak{F}_{e}$ of fragments of $e$. Obviously, every Riesz subspace of $L_{0}(\mu)$ for a finite measure $\mu$ is measurable. The aim of the present paper is to show that the above example is quite general. In our proofs, we use and develop a new technique proposed in recent paper [5].

For familiarly used terminology and notation, we refer the reader to the textbook [2]. An element $x$ of a Riesz space $E$ is said to be a fragment ${ }^{1}$ of another element $y \in E$ provided $x \perp(y-x)$. We write $x \sqsubseteq y$ to express that $x$ is a fragment of $y$. It is not hard to see that $\sqsubseteq$ is a partial order on $E$, called the lateral order, which coincides with the usual order $\leq$ on $E^{+}$. The lateral supremum (or infimum) of a subset $G \subseteq E$, that is, the supremum (respectively, infimum) with respect to the order $\sqsubseteq$, is denoted by $\bigcup G$ (respectively, $\cap G$ ). For more information on the lateral order see [5]. The notation $z=x \sqcup y$ for elements $x, y, z$ of $E$ means that $z=x+y$ and $x \perp y$. For instance, if $x \sqsubseteq y$ then $y=x \sqcup(y-x)$ is a decomposition to disjoint fragments. The set $\mathfrak{F}_{e}$ of all fragments of an element $e \in E \backslash\{0\}$ is a Boolean algebra with respect to the operations of taking the lateral supremum and infimum, zero 0 and unit $e$ (see [2, Theorem 3.15] for a positive $e$ and [5, Proposition 3.4] for an arbitrary $e$ ). Moreover, $\mathfrak{F}_{e}$

$\mathrm{\textrm {y } \triangle \mathrm { K } 5 1 7 . 9 8 2}$

2020 Mathematics Subject Classification: primary 46A40, secondary $46 \mathrm{E} 30$.

${ }^{1}$ component, in the terminology of [2]. 
is the lateral ideal and the lateral band, generated by $e$ (see [4] for details).

We say that a net $\left(x_{\alpha}\right)_{\alpha \in A}$ in a Riesz space E order converges to a limit $x \in E$ if there is a net $\left(y_{\alpha}\right)_{\alpha \in A}$ in $E$ such that $y_{\alpha} \downarrow 0$ and $\left|x_{\alpha}-x\right| \leq y_{\alpha}$ for some $\alpha_{0} \in A$ and all $\alpha \geq \alpha_{0}$ (write $x_{\alpha} \stackrel{o}{\longrightarrow} x$ ). Remark that there is another notion of order convergence in the literature, where the index set of the majorizing net $\left(y_{\alpha}\right)$ can be different from $A$, however for our purposes these two notions coincide (for laterally increasing nets, on the one hand, and for the notion of order continuity for regular operators [1, Theorem 1.7], on the other hand).

Recall that a Riesz space $E$ is said to be $C$-complete if every nonempty laterally bounded subset $G$ of $E$ has a lateral supremum $\bigcup G \in E$. If a Riesz space $E$ is either Dedekind complete or laterally complete then $E$ is $C$-complete [5, Corollary 5.8]. The Banach lattice $C[0,1]$ is a $\mathrm{C}$-complete Riesz space which is neither Dedekind complete, nor laterally complete.

We do not know whether there is an atomless example of the kind. Note that some results on orthogonally additive operators on C-complete Riesz spaces were established in recent paper [7].

We say that a net $\left(x_{\alpha}\right)_{\alpha \in A}$ in a Riesz space $E$ horizontally converges to an element $x \in E$ and $x$ is a horizontal limit of $\left(x_{\alpha}\right)$ and write $x_{\alpha} \stackrel{h}{\longrightarrow} x$ provided $\left(x_{\alpha}\right)$ is laterally increasing, that is, $x_{\alpha} \sqsubseteq x_{\beta}$ as $\alpha<\beta$, and $\bigcup_{\alpha \in A} x_{\alpha}=x$ (equivalently, $x_{\alpha} \stackrel{o}{\longrightarrow} x$ ). The horizontal closure $\bar{G}^{h}$ (or horizontal $\sigma$-closure $\bar{G}^{h \sigma}$ ) of a subset $G$ of $E$ is defined to be the set of all horizontal limits of nets (respectively, sequences) from $G$. A nonempty subset $G$ of $E$ is said to be horizontally closed (or horizontally $\sigma$-closed) if $\bar{G}^{h}=G$ (respectively, $\bar{G}^{h \sigma}=G$ ).

Definition 1. A solid subset of a Riesz space $E$ which is closed under multiplication by scalars will be called a pre-ideal of $E$.

Obviously, every ideal of $E$ is a pre-ideal of $E$. The set-theoretical union of two nontrivial disjoint ideals of $E$ provides a simple example of a pre-ideal which is not an ideal.

\section{Measurable elements and the measurable part of a Riesz space}

Recall that a Boolean algebra $\mathcal{B}$ is said to be measurable provided $\mathcal{B}$ is Dedekind $\sigma$-complete and there is a positive probability measure $\mu: \mathcal{B} \rightarrow[0,1]$, that is, a function possessing the following properties:

$$
\begin{aligned}
& \mu(\mathbf{1})=1 ; \\
& \mu(x)>0 \text { for all } x \in \mathcal{B} \backslash\{\mathbf{0}\} ; \\
& \mu\left(\sup _{n} x_{n}\right)=\sum_{n=1}^{\infty} \mu\left(x_{n}\right) \text { for every disjoint sequence }\left(x_{n}\right)_{n=1}^{\infty} \text { in } \mathcal{B} .
\end{aligned}
$$

Definition 2. Let $E$ be a Riesz space. We say that an element $e \in E \backslash\{0\}$ is measurable if the Boolean algebra $\mathfrak{F}_{e}$ of all fragments of $e$ is measurable.

Recall that an element $e \neq 0$ of $E$ is an atom if $\mathfrak{F}_{e}=\{0, e\}$ (actually, the original definition of an atom differs from the given one, however the definitions are equivalent if $E$ has the principal projection property [6, Proposition 1.3]).

Definition 3. Given a Riesz space $E$, by the measurable part of $E$ we mean the set $E_{\text {meas }}$ of all measurable elements of $E$ together with zero. 
To prove the following statement is an easy exercise.

Proposition 1. Let E be a Riesz space. Then the following assertions hold.

(i) If $a$ is an atom of $E$ then $a \in E_{\text {meas. }}$.

(ii) For every $x, y \in E$, if $x \in E_{\text {meas }}$ and $y \sqsubseteq x$ then $y \in E_{\text {meas. }}$.

(iii) For every $x, y \in E_{\text {meas, }}$ if $x \perp y$ then $x+y \in E_{\text {meas. }}$.

(iv) For every $x, y \in E$ with $|x|=|y|$, the conditions $x \in E_{\text {meas }}$ and $y \in E_{\text {meas }}$ are equivalent.

Theorem 1. Let $E$ be a Riesz space with the principal projection property. Then $E_{\text {meas }}$ is a $\sigma$-ideal of $E$.

Although the idea of proof is clear, it needs some technical efforts to prove that $E_{\text {meas }}$ is solid. In passing, we prove some lemmas which may have an independent interest. The first one shows that in some cases the order convergence and order closure can be replaced with the horizontal convergence and horizontal closure.

Lemma 1. Let $E$ be a Riesz space with the principal projection property, I a pre-ideal of $E$, $\left(x_{\alpha}\right)_{\alpha \in A}$ a net in I and $x \in E$. If $0 \leq x_{\alpha} \uparrow x$ then there exists a net $\left(y_{\alpha}\right)_{\alpha \in A}$ in I with $y_{\alpha} \stackrel{h}{\longrightarrow} x$.

Proof. Set $u_{\alpha}=\left(2 x_{\alpha}-x\right)^{+}$and $y_{\alpha}=P_{u_{\alpha}} x$ for all $\alpha \in A$ and show that $\left(y_{\alpha}\right)_{\alpha \in A}$ possesses the desired properties. By the definitions, $y_{\alpha} \sqsubseteq x$ for all $\alpha \in A$. Since $x_{\alpha} \uparrow$, one has $u_{\alpha} \uparrow$ and hence $y_{\alpha} \uparrow$. Observe that $u_{\alpha} \stackrel{o}{\longrightarrow}(2 x-x)^{+}=x$. Hence, by [2, Theorem 1.48], $y_{\alpha}=P_{u_{\alpha}} x \stackrel{o}{\longrightarrow} P_{x} x=x$. Thus, $y_{\alpha} \stackrel{h}{\longrightarrow} x$. It remains to show that $y_{\alpha} \in I$ for all $\alpha$. Indeed, for any index $\alpha$ one has

$$
\begin{aligned}
P_{u_{\alpha}}\left(2 x_{\alpha}\right)-y_{\alpha}=P_{u_{\alpha}}\left(2 x_{\alpha}-x\right) & =P_{\left(2 x_{\alpha}-x\right)^{+}}\left(\left(2 x_{\alpha}-x\right)^{+}-\left(2 x_{\alpha}-x\right)^{-}\right) \\
& =P_{\left(2 x_{\alpha}-x\right)^{+}}\left(2 x_{\alpha}-x\right)^{+}=P_{u_{\alpha}} u_{\alpha}=u_{\alpha} \geq 0,
\end{aligned}
$$

which implies $\left|y_{\alpha}\right|=y_{\alpha} \leq P_{u_{\alpha}}\left(2 x_{\alpha}\right) \leq 2 x_{\alpha} \in I$. Since $I$ is solid, $y_{\alpha} \in I$.

Using [2, Lemma 1.37], we obtain the following consequence of Lemma 1.

Corollary 1. Let $E$ be a Riesz space with the principal projection property and $A$ a pre-ideal of $E$. Then the order closure of $A$ equals the horizontal closure of $A$, and the order $\sigma$-closure of $A$ equals the horizontal $\sigma$-closure of $A$.

Using standard terminology, by a $\sigma$-ideal of a Riesz space $E$ we mean a $\sigma$-order closed ideal of $E$. Below is another consequence of Lemma 1.

Corollary 2. Let I be an ideal of a Riesz space $E$ with the principal projection property. Then the band generated by I equals the horizontal closure $\bar{I}^{h}$ of $I$, and the $\sigma$-ideal generated by $I$ equals the horizontal $\sigma$-closure $\bar{I}^{h \sigma}$ of I. Hence, every horizontally closed ideal is a band and every horizontally $\sigma$-closed ideal is a $\sigma$-ideal.

Lemma 2. Let I be a pre-ideal of a Riesz space $E$ with the principal projection property. If I is closed under disjoint sums then $I$ is an ideal. 
Proof. Fix any $0 \leq x, y \in I$. It is enough to show that $x+y \in I$. Set $u:=P_{(y-x)^{+}}(x+y)$ and $v:=x+y-u$. Since $u \sqsubseteq x+y$, one has $x+y=u \sqcup v$. Our goal is to show that $u, v \in I$. Observe that $(y-x)^{+} \perp(y-x)^{-}$implies $P_{(y-x)^{+}}(y-x)^{-}=0$. Hence, $P_{(y-x)^{+}}(y-x)=$ $P_{(y-x)^{+}}(y-x)^{+}=(y-x)^{+} \geq 0$, which yields $P_{(y-x)^{+}} x \leq P_{(y-x)^{+}} y \leq y$. Therefore,

$$
0 \leq u=P_{(y-x)^{+}}(x+y)=P_{(y-x)^{+}} x+P_{(y-x)+} y \leq 2 P_{(y-x)^{+}} y \leq 2 y
$$

and analogously,

$$
P_{(y-x)^{-}}(x+y) \leq 2 x \text {. }
$$

Then

$$
\begin{gathered}
0 \leq v=P_{x+y}(x+y)-u \stackrel{\text { by }}{\stackrel{[1, p .39]}{=} P_{-(y-x)^{-}}(x+y)-P_{(y-x)^{+} \wedge(y-x)^{-}}(x+y)} \\
\leq P_{-(y-x)^{-}}(x+y) \stackrel{\text { by (2) }}{\leq} 2 x .
\end{gathered}
$$

Since $x, y \in I$, by (1) and (3) we obtain $u, v \in I$.

Now Corollary 2 and Lemma 2 imply the following lemma.

Lemma 3. Let I be a horizontally closed (or horizontally $\sigma$-closed) pre-ideal in a Riesz space $E$ with the principal projection property, closed under disjoint sums. Then $I$ is a band (respectively, $\sigma$-ideal) in $E$.

Proof of Theorem 1. We prove that $B:=E_{\text {meas }}$ satisfies the assumptions of Lemma 3. First we prove that $B$ is solid. Assume first $x \in E, e \in B$ and $0<x \leq e$ and prove that $x \in B$. First show that $\mathfrak{F}_{x}$ is Dedekind $\sigma$-complete. Given any sequence $x_{n} \uparrow$ in $\mathfrak{F}_{x}$, we set $e_{n}=P_{x_{n}}$ efor all $n \in \mathbb{N}$. Obviously, $e_{n} \uparrow$ in $\mathfrak{F}_{e}$. Since $e_{n} \in \mathfrak{F}_{e}$ and $\mathfrak{F}_{e}$ is Dedekind $\sigma$-complete, there exists $e_{0} \in \mathfrak{F}_{e}$ such that $e_{n} \uparrow e_{0}$.

Show that $x_{n}=P_{e_{n}} x$ for all $n \in \mathbb{N}$. Since $x_{n} \sqsubseteq x$, for every $i \in \mathbb{N}$ one has $x=x_{n} \vee\left(x-x_{n}\right)$ and hence $x \wedge i x_{n}=\left(x_{n} \wedge i x_{n}\right) \vee\left(\left(x-x_{n}\right) \wedge i x_{n}\right)=x_{n} \vee 0=x_{n}$. Therefore, for every $n, k, m \in$ $\mathbb{N}$ one has

$$
k e \wedge x \wedge k m x_{n}=k e \wedge x_{n}=x_{n} .
$$

Then by [2, Theorem 1.47] and the infinite distributive law, for every $n \in \mathbb{N}$

$$
P_{e_{n}} x=\bigvee_{k=1}^{\infty}\left(x \wedge k e_{n}\right)=\bigvee_{k=1}^{\infty}\left(x \wedge k \bigvee_{m=1}^{\infty}\left(e \wedge m x_{n}\right)\right)=\bigvee_{k=1}^{\infty} \bigvee_{m=1}^{\infty}\left(x \wedge k e \wedge k m x_{n}\right) \stackrel{(4)}{=} x_{n}
$$

Set $x_{0}=P_{e_{0}} x \in \mathfrak{F}_{x}$. Since $e_{n} \uparrow e_{0}$, by [2, Theorem 1.48],

$$
x_{n} \stackrel{(5)}{=} P_{e_{n}} x \uparrow P_{e_{0}} x=x_{0} .
$$

Now consider the general case, $x \in E, e \in B$ and $0<|x| \leq|e|$. Then the conclusion $x \in B$ follows from the above particular case and (iv) of Proposition 1. So, the Dedekind $\sigma$-completeness of $\mathfrak{F}_{x}$ is proved.

Let $\mu_{e}: \mathfrak{F}_{e} \rightarrow[0,1]$ be a positive probability measure. We define a function $\mu_{x}: \mathfrak{F}_{x} \rightarrow[0,1]$ by setting $\lambda=\left(\mu_{e}\left(P_{x}(e)\right)\right)^{-1}$ and $\mu_{x}(y)=\lambda \mu_{e}\left(P_{y}(e)\right)$ for all $y \in \mathfrak{F}_{x}$ and show that $\mu_{x}$ is a positive probability measure on $\mathfrak{F}_{x}$. Obviously, $\mu_{x}(x)=1$. Let $0<y \in \mathfrak{F}_{x}$. Then

$$
P_{y} e=\bigvee_{n=1}^{\infty}(e \wedge n y) \geq e \wedge y=y>0
$$


and hence, $\mu_{e}\left(P_{y}(e)\right)>0$. So, the function $\mu_{x}$ is strictly positive. Now we show the $\sigma$-additivity of $\mu_{x}$. Let $\left(x_{n}\right)_{n=1}^{\infty}$ be a disjoint sequence in $\mathfrak{F}_{x}$. Set $u_{n}=\bigvee_{k=1}^{n} x_{k}$ for all $n \in \mathbb{N}$ and $u=\bigvee_{n=1}^{\infty} x_{n}$. Since $u_{n} \uparrow u$, by [2, Theorem 1.48],

$$
\mu_{x}\left(u_{n}\right)=\lambda \mu_{e}\left(P_{u_{n}}(e)\right) \uparrow \lambda \mu_{e}\left(P_{u}(e)\right)=\mu_{x}(u) .
$$

On the other hand,

$$
\mu_{x}\left(u_{n}\right)=\lambda \mu_{e}\left(P_{u_{n}}(e)\right)=\lambda \mu_{e}\left(\bigsqcup_{k=1}^{n} P_{x_{k}}(e)\right)=\sum_{k=1}^{n} \lambda \mu_{e}\left(P_{x_{k}}(e)\right)=\sum_{k=1}^{n} \mu_{x}\left(x_{k}\right) .
$$

By (6) and (7), $\sum_{n=1}^{\infty} \mu_{x}\left(x_{n}\right)=\mu_{x}(u)$ and the $\sigma$-additivity of $\mu_{x}$ is proved. Thus, $x \in B$ and so, $B$ is solid.

Obviously, $B$ is closed under multiplication by a scalar, and so, $B$ is a pre-ideal. By (iii) of Proposition $1, B$ is closed under disjoint sums. It remains to show that $B$ is horizontally $\sigma$-closed. Let $x \in E, x_{n} \in B$ for $n \in \mathbb{N}$ and $x_{n} \stackrel{h}{\longrightarrow} x$. Show that $x \in B$. Set $y_{1}=x_{1}$ and $y_{n}=x_{n}-x_{n-1}$ for $n>1$. Then $\left(y_{n}\right)_{n=1}^{\infty}$ is a disjoint sequence with $x=\bigcup_{n=1}^{\infty} y_{n}$ and $y_{n} \in B$ by (ii) of Proposition 1 . Let for every $n \in \mathbb{N}, \mu_{n}: \mathfrak{F}_{y_{n}} \rightarrow[0,1]$ be a positive probability measure. Then the formula

$$
\mu(z)=\sum_{n=1}^{\infty} 2^{-n} \mu_{n}\left(z \cap y_{n}\right), \quad z \in \mathfrak{F}_{x}
$$

defines a positive probability measure $\mu: \mathfrak{F}_{x} \rightarrow[0,1]$ and so, $x \in B$.

The following simple example shows that the measurable part need not be a band in a Dedekind complete Banach lattice.

Example 1. Let $E=\ell_{\infty}(\Gamma)$ for an uncountable set $\Gamma$. Then

$$
E_{\text {meas }}=\left\{x \in \ell_{\infty}(\Gamma):|\operatorname{supp} x| \leq \aleph_{0}\right\},
$$

which is not a band of $E$.

Definition 4. Say that a Riesz space $E$ is

measurable provided all elements of $E$ are measurable, that is, $E_{\text {meas }}=E$;

strictly measurable provided E has a measurable weak unit.

To show that strict measurability implies measurability, we need the following simple observation.

Proposition 2. Let $E$ be a Riesz space. Then for every $e \in E$ the $\sigma$-ideal generated by e equals the principal band $B_{e}$ generated by $e$.

Proof. Denote by $X_{e}$ the $\sigma$-ideal generated by e. Obviously, $E_{e} \subseteq X_{e} \subseteq B_{e}$. By [2, Theorem 1.38], $B_{e}=\{x \in E:|x| \wedge n|e| \uparrow|x|\}$, and hence, every $x \in B_{e}$ equals an order limit of a sequence from $E_{e}$, and thus, $x \in X_{e}$.

As a consequence of Theorem 1 and Proposition 2 we obtain the following statement. 
Proposition 3. Let E be a strictly measurable Riesz space with the principal projection property. Then $E$ is measurable.

Example 2. Let $E=c_{0}(\Gamma)$ for an uncountable set $\Gamma$. Then $E$ is measurable but not strictly measurable.

Recall that a Riesz space is said to have the countable chain condition (CCC, in short) provided every disjoint set in $E$ is at most countable.

Proposition 4. A strictly measurable Riesz space with the principal projection property possesses the countable chain condition.

Proof. Let $D$ be a disjoint subset of $E$ and $e>0$ be a measurable weak unit of $E$. For every $d \in D$ we set $e_{d}=P_{d} e$. Then $e_{d} \in \mathfrak{F}_{e}$ and $e_{d}>0$, because $e$ is a weak unit. Moreover, if $d^{\prime} \neq d^{\prime \prime}$ then $e_{d^{\prime}} \perp e_{d^{\prime \prime}}$ and hence $e_{d^{\prime}} \neq e_{d^{\prime \prime}}$. Being a disjoint subset of the measurable Boolean algebra $\mathfrak{F}_{e}$, the family $\left(e_{d}\right)_{d \in D}$ is at most countable, and so is $D$.

\section{Measurable Riesz spaces with the CCC}

Following Fremlin [3], a band $B$ of a Riesz space is said to be complemented provided $B^{\perp \perp}=B$. So, every projection band $B$ (that is, $E=B \oplus B^{\perp}$ ) is complemented. For every Riesz space $E$ the set $\mathcal{U}$ of all complemented bands of $E$ is a Dedekind complete Boolean algebra with respect to $B^{\prime} \wedge_{\mathcal{U}} B^{\prime \prime}=B^{\prime} \cap B^{\prime \prime}, B^{\prime} \vee_{\mathcal{U}} B^{\prime \prime}=\left(B^{\prime}+B^{\prime \prime}\right)^{\perp \perp}, \mathbf{1}_{\mathcal{U}}=E, \mathbf{0}_{\mathcal{U}}=\{0\}$, $\mathbf{1}_{\mathcal{U}} \backslash \mathcal{U} B=B^{\perp}, B^{\prime} \leq_{\mathcal{U}} B^{\prime \prime} \Leftrightarrow B^{\prime} \subseteq B^{\prime \prime}[3,352 Q]$. If a Riesz space is Archimedean (which is the case in every our statement) then every band of $E$ is complemented [3,353B]. So, for an Archimedean Riesz space $E$ we are going to consider the Boolean algebra $\mathcal{U}$ of all bands of $E$, which is Dedekind complete by the above theorem.

We need the following result on embedding Riesz spaces in $L_{0}(\mathcal{B})$, where $\mathcal{B}$ is a Boolean algebra (for the definition of the Riesz space $L_{0}(\mathcal{B})$ see $[3,364]$ ).

Theorem 2 ([3, 368E]). Let $E$ be an Archimedean Riesz space and $\mathcal{U}$ its band Boolean algebra. Then $E$ can be embedded as an order dense Riesz subspace of $L_{0}(\mathcal{U})$.

Using Theorem 2, we obtain necessary and sufficient conditions on a Riesz space with the principal projection property to have measurable Boolean algebra of bands.

Theorem 3. Let $E$ be a Riesz space with the principal projection property. Then the following assertions are equivalent.

1. The Boolean algebra $\mathcal{U}$ of bands of $E$ is measurable.

2. E is measurable and satisfies the CCC.

3. E can be embedded as an order dense subspace of $L_{0}(\mu)$ for some probability measure $\mu$.

For the proof we need the following lemma.

Lemma 4. Let $E$ be a $C$-complete Riesz space with a principal projection property and a weak unit $e>0$. Then every band $B$ of $E$ is a principal band $B_{\tau(B)}$ generated by some unique fragment $\tau(B) \sqsubseteq e$. Moreover, the defined above function $\tau: \mathcal{U} \rightarrow \mathfrak{F}_{e}$ preserves disjointness, where $\mathcal{U}$ is the Boolean algebra of bands of $E$. 
Before the proof we recall that the set-theoretical difference $x \backslash y$ for fragments $x, y$ of an element $e \in E$ is defined by setting $x \backslash y=x \cap(e-y)$ and equals $x \backslash y=x-x \cap y$ (see [5] for the details).

Proof. Let $B \in \mathcal{U}$. Define a vector $e^{*} \in \mathfrak{F}_{e}$ by setting

$$
e^{*}=\bigcup\left(\mathfrak{F}_{e} \cap B\right)
$$

(the lateral supremum in (8) exists by the $C$-completeness of $E$, because the set $\mathfrak{F}_{e} \cap B$ contains zero and therefore is nonempty, and is laterally bounded from above by $e$ ).

Show that $B=B_{e^{*}}$. Suppose $x \in B$ and prove that $x \in B_{e^{*}}$. With no loss of generality we may and do assume that $x \geq 0$. Since $x \in E=B_{e}$, we have $x \wedge n e \uparrow x$. By [2, Theorem 1.47], $P_{x} e=\bigvee_{m=1}^{\infty}(e \wedge m x)$. Observe that $P_{x} e \in \mathfrak{F}_{e} \cap B$ and by (8), $P_{x} e \leq e^{*}$. Hence, using the distributivity law $[2$, Theorem 1.8], for every $n \in \mathbb{N}$ we obtain

$$
x \wedge n e=\bigvee_{m=1}^{\infty}(x \wedge n e)=\bigvee_{m=1}^{\infty}(x \wedge n(e \wedge m x))=x \wedge n \bigvee_{m=1}^{\infty}(e \wedge m x)=x \wedge n P_{x} e \leq x \wedge n e^{*} \in B_{e^{*}}
$$

This yields $x \wedge n e \uparrow x \in B_{e^{*}}$.

Now suppose $x \in B_{e^{*}}$ and prove that $x \in B$. Again we assume additionally that $x \geq 0$. By the assumptions, $x \wedge n e^{*} \uparrow x$. Then $x \wedge n e^{*} \in B$ and hence, $x \in B$.

Show the uniqueness of the fragment $e^{*} \sqsubseteq e$ such that $B=B_{e^{*}}$. Let $B_{x}=B_{y}$ for some $x, y \in F_{e}$. If $x \neq y$ then either $x \cap y \neq 0$ or $y \cap x \neq 0$ [5, Proposition 3.18]. Say, $z:=x \cap y \neq 0$. Then $z \in B_{x} \backslash B_{y}$, a contradiction.

It remains to show that $\tau$ preserves disjointness. Let $B^{\prime}, B^{\prime \prime}$ be disjoint bands of $E$. In terms of $\tau$, this means that $B_{\tau\left(B^{\prime}\right)} \cap B_{\tau\left(B^{\prime \prime}\right)}=B^{\prime} \cap B^{\prime \prime}=\{0\}$. Set $z:=\tau\left(B^{\prime}\right) \cap \tau\left(B^{\prime \prime}\right)$. Since $x \in B_{x}$ for all $x \in E$, we obtain $z \in B_{\tau\left(B^{\prime}\right)} \cap B_{\tau\left(B^{\prime \prime}\right)}=\{0\}$, which yields $z=0$, and so $\tau\left(B^{\prime}\right)$ and $\tau\left(B^{\prime \prime}\right)$ are disjoint elements of $\mathfrak{F}_{e}{ }^{2}$

Corollary 3. Let E be a Dedekind complete Riesz space with a weak unit. Then every band of $E$ is a principal band.

Corollary 4. Let $E$ be a laterally complete Riesz space. Then every band of $E$ is a principal band.

Proof. It is enough to prove that every laterally complete Riesz space has a weak unit. The latter statement is easy to prove by showing that the lateral supremum of any maximal disjoint set is a weak unit.

Proof of Theorem 3. (1) $\Rightarrow$ (3) follows from Theorem 2 .

(3) $\Rightarrow(2)$. Let $J: E \rightarrow L_{0}(\mu)$ be an order $\sigma$-continuous lattice homomorphism for some probability measure $\mu$. Since $L_{0}(\mu)$ satisfies the CCC, so does $E$. Show that $E$ is measurable. Given any $e \in E^{+} \backslash\{0\}$ and $x \in \mathfrak{F}_{e}$, we set

$$
\bar{\mu}(x)=\frac{\mu(\operatorname{supp} J x)}{\mu(\operatorname{supp} J e)} .
$$

${ }^{2}$ we draw reader's attention that $\cap$ means the lateral supremum in the definition of $z$, while $\cap$ means the settheoretical intersection in the other places of the paragraph. 
It is immediate that $\bar{\mu}: \mathfrak{F}_{e} \rightarrow[0,1]$ is a probability measure. So, $e$ is measurable.

$(2) \Rightarrow(1)$. Let $D$ be a maximal disjoint subset of $E$, which is at most countable by the assumption. Let $\left(a_{d}\right)_{d \in D}$ be numbers $a_{d}>0$ with $\sum_{d \in D} a_{d}=1$. Given any $d \in D$, let $\mu_{d}: \mathfrak{F}_{d} \rightarrow[0,1]$ be a positive probability measure. Then we define a positive probability measure $\mu: \mathcal{U} \rightarrow[0,1]$ by setting

$$
\mu(B)=\sum_{d \in D} \mu_{d}\left(\tau_{d}\left(B \cap B_{d}\right)\right), B \in \mathcal{U},
$$

where $t=\tau_{d}\left(B \cap B_{d}\right)$ is the element of $\mathfrak{F}_{d}$ such that $B \cap B_{d}=B_{t}$ (see Lemma 4 ). We omit a straightforward demonstration that (9) defines a positive probability measure.

\section{Acknowledgements}

Marat Pliev was supported by the Russian Foundation for Basic Research (grant number 17-51-12064).

\section{References}

[1] Abramovich Yu., Sirotkin G. On order convergence of nets. Positivity 2005, 9 (3), 287-292. doi:10.1007/s11117004-7543-x

[2] Aliprantis C.D., Burkinshaw O. Positive Operators. Springer, Dordrecht, 2006.

[3] Fremlin D.H. Measure Theory. Vol. 3. Measure algebras. Torres Fremlin, Colchester, 2004.

[4] Kamińska A., Krasikova I., Popov M. Projection lateral bands and lateral retracts. Carpathian Math. Publ. 2020, 12 (2), 333-339. doi:10.15330/cmp.12.2.333-339

[5] Mykhaylyuk V., Pliev M., Popov M. The lateral order on Riesz spaces and orthogonally additive operators. Positivity 2021, 25 (2), 291-327. doi:10.1007/s11117-020-00761-x

[6] Mykhaylyuk V., Pliev M., Popov M., Sobchuk O. Dividing measures and narrow operators. Studia Math. 2015, 231 (2), 97-116. doi:10.4064/sm7878-2-2016

[7] Pliev M. On C-compact orthogonally additive operators. J. Math. Anal. Appl. 2021, 494 (1), 124594. doi: 10.1016/j.jmaa.2020.124594

[8] Popov M. Horizontal Egorov property of Riesz spaces. Proc. Amer. Math. Soc. 2021, 149 (1), 323-332. doi: $10.1090 / \mathrm{proc} / 15235$

Received 26.10.2020

Красікова І., Плієв М., Попов М. Вимірні векторні гратки // Карпатські матем. публ. - 2021. — T.13, №1. - C. 81-88.

Ми досліджуємо вимірні елементи векторної гратки $E$, тобто такі елементи $e \in E \backslash\{0\}$, для яких булева алгебра $\mathfrak{F}_{e}$ фррагментів елемента $е \in$ вимірною. Зокрема, ми доводимо, що множина $E_{\text {meas }}$ всіх вимірних елементів векторної гратки $E$, що має головну проективну властивість, разом із нулем утворює $\sigma$-ідеал в $Е$. Інший результат стверджує, що для довільної векторної гратки $E$ з головною проективною властивістю наступні умови еквівалентні.

(1) Булева алгебра $\mathcal{U}$ всіх смуг в $E \in$ вимірною.

(2) $E_{\text {meas }}=E$ та $E$ задовольняє умову зліченності ланцюгів.

(3) Векторна гратка $Е$ ізоморфно вкладається, як порядково щільна підгратка векторної гратки $L_{0}(\mu)$ для деякої ймовірнісної міри $\mu$.

Ключові слова і фрази: векторна гратка, булева алгебра смуг. 